\title{
Cerebellar liponeurocytoma with atypical histological features - a rare example of a glioneuronal tumor
}

\author{
Blanka Hermann ${ }^{1,2}$, Michal Woznica ${ }^{3}$, Wojciech Kloc $^{3,4}$, Piotr Borkowski ${ }^{5}$, Witold Libionka ${ }^{3,6}$, Ewa Izycka-Swieszewska ${ }^{1,2}$ \\ ${ }^{1}$ Department of Pathology and Neuropathology, Medical University in Gdansk, ${ }^{2}$ Department of Pathomorphology, Copernicus \\ Hospitals, Gdansk, ${ }^{3}$ Department of Neurosurgery, Copernicus Hospitals, Gdansk, ${ }^{4}$ Department of Neurology and Neurosurgery, \\ University of Warmia and Mazury, ${ }^{5}$ Department of Radiology, Copernicus Hospitals, Gdansk, ${ }^{6}$ Department of Physiotherapy, \\ Faculty of Rehabilitation and Kinesiology, Gdansk University of Physical Education and Sport, Gdansk, Poland
}

\begin{abstract}
We present a case of a rare neoplasm in a 77-year-old woman with previous oncological history, who developed a rapid onset of cerebellar symptoms. The neuroimaging detected a posterior fossa tumor suspected of meningioma which was completely resected soon after. Histologically the neoplasm had two components with different immunophenotype. One constituent was lobular, composed of monotonous mitotically active round cells with a predominant neuronal profile. The second, astrocytic component contained lipomatous cells intermixed with larger gemistocytic astrocytes. Fields of geographic necrosis as well as multifocal microvascular proliferation were observed. The Ki67 proliferation index was $12 \%$. After two years of follow-up the patient remains free of symptoms and radiologic recurrence. The presented case of cerebellar liponeurocytoma is unusual in terms of its atypical histological features and prominent astrocytic component. The authors propose that the term 'lipomatous glioneuronal tumor' seems to be more appropriate for this type of lesion, considering its histologic spectrum and possible extracerebellar location.
\end{abstract}

Key words: cerebellar liponeurocytoma, lipidized tumor, glioneuronal tumor.

\section{Introduction}

Cerebellar liponeurocytoma is a rare and poorly understood neoplasm with a distinctive morphology and generally indolent behavior. It was first described in 1978 by Bechtel et al. [2], and nearly 50 cases under several synonyms have been reported since $[11,26,32,33]$. In the 2000 World Health Organization (WHO) classification of Tumors of the Central Nervous System it was categorized for the first time as a distinct entity within the neuronal and mixed neu- ronal-glial tumors section. In 2016, the WHO defined it as a rare cerebellar neoplasm with advanced neuronal/neurocytic differentiation and focal lipoma-like changes [22]. The prognosis of cerebellar liponeurocytoma is difficult to assess given the low number of described cases. Most patients survive more than 5 years, but recurrences concern almost $50 \%$ of the patients [20]. Moreover, a few reports of aggressive behavior of liponeurocytomas with fast regrowth and poor outcome have been published [16,24]. This lesion develops mainly in the cerebellum, but it can 

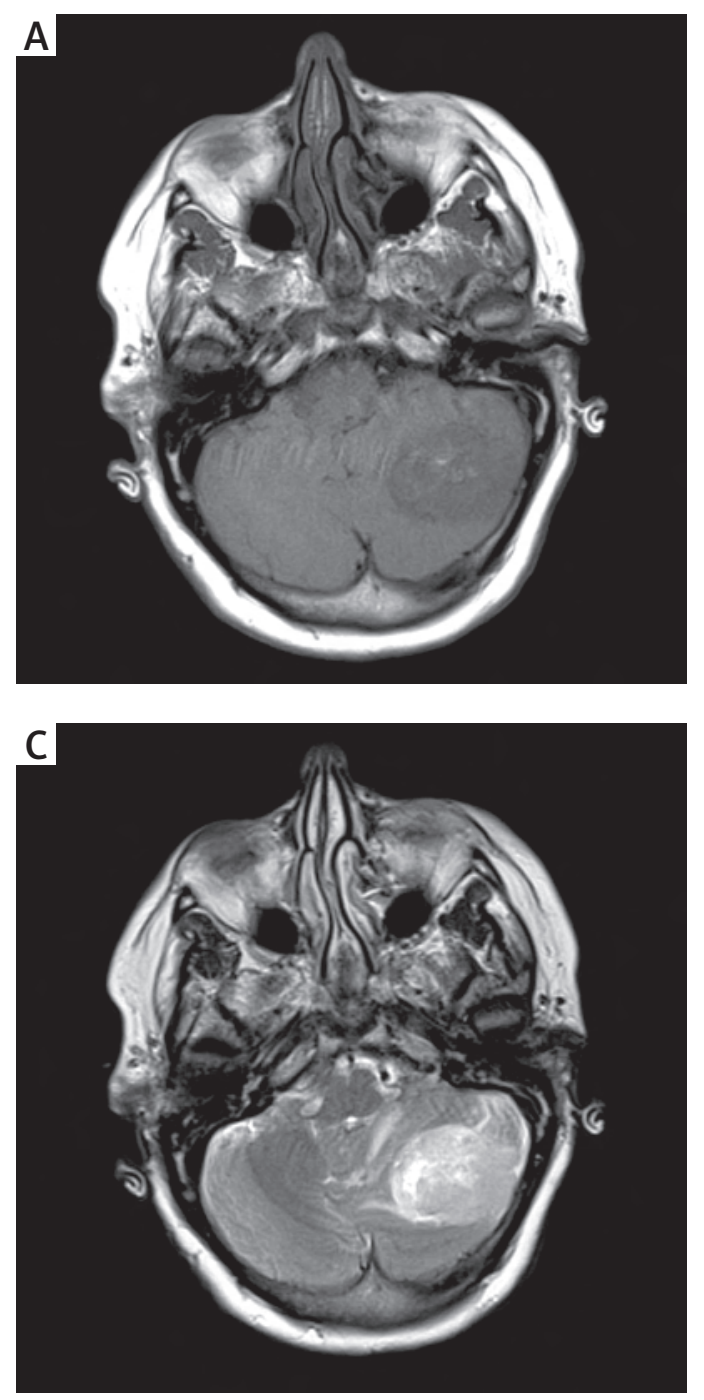
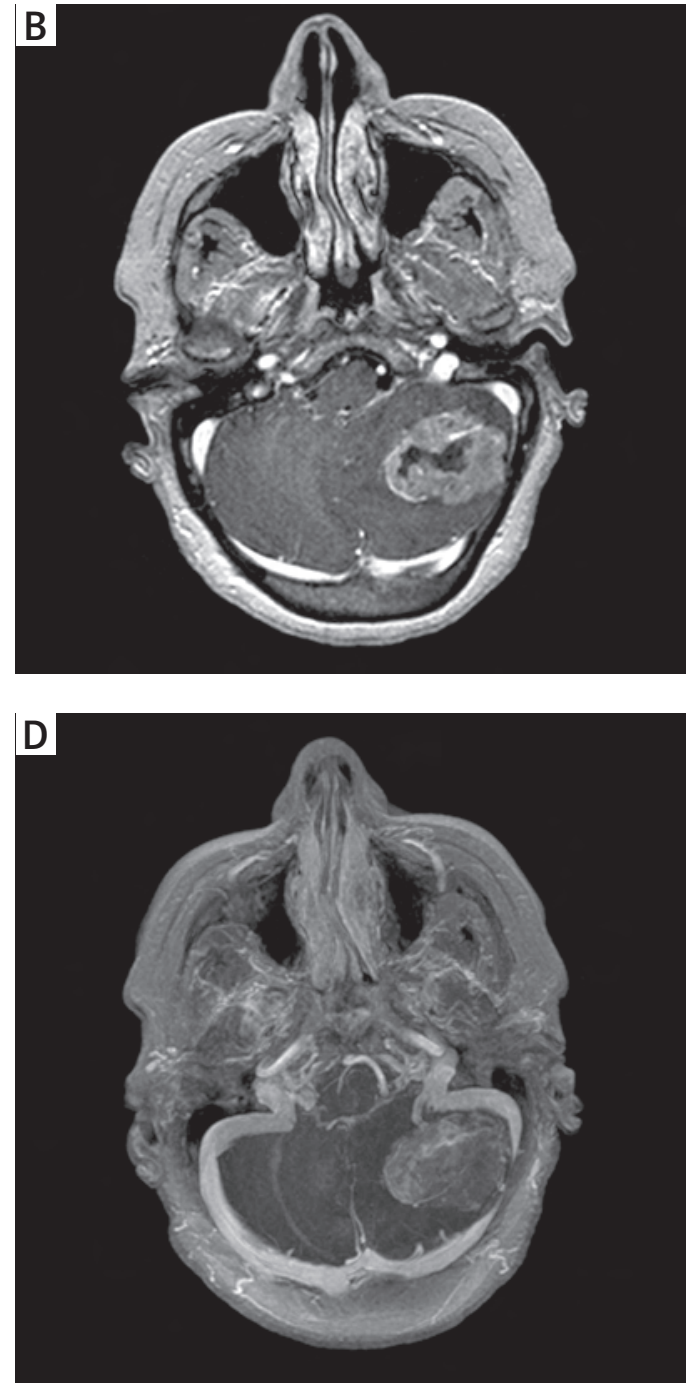

Fig. 1. Magnetic resonance imaging axial views demonstrated mostly well-delineated heterogeneous mass within cerebellar hemisphere measuring about $4 \times 3.5 \times 2.5 \mathrm{~cm}$. The tumor widely adhered to the dura and there was minimal perilesional edema. On T1-weighted images (A) the tumor was hypointense with hyperintense areas. After contrast injection (B) the mass was moderately enhancing and on T2-weighted views (C) it was hyperintense to the cerebellum. D) 3D reconstruction.

also occur supratentorially. Single cases suggesting familial predisposition for such tumors have been published recently $[26,33]$.

\section{Case report}

\section{Clinical description}

A 77-year-old woman was admitted to the neurosurgery department in April 2015 with a threeweek history of dizziness, headaches and nausea. The patient had a history of colorectal cancer stage T2NOMO treated with hemicolectomy in 2008, and a spinal thoracic schwannoma excised totally in 2013. Her central nervous system (CNS) radiological follow-up was uneventful in 2014. Due to a sudden onset of neurological symptoms, she was suspected of cerebral metastasis of colorectal cancer; however, computed tomography (CT) examination showed posterior fossa tumor suggesting meningioma. Moreover, the patient suffered from stable coronary artery disease and hypercholesterolemia. At admission she was conscious with good contact, with slightly unsteady gait and positive finger-nose test and Romberg test on neurological examination. 

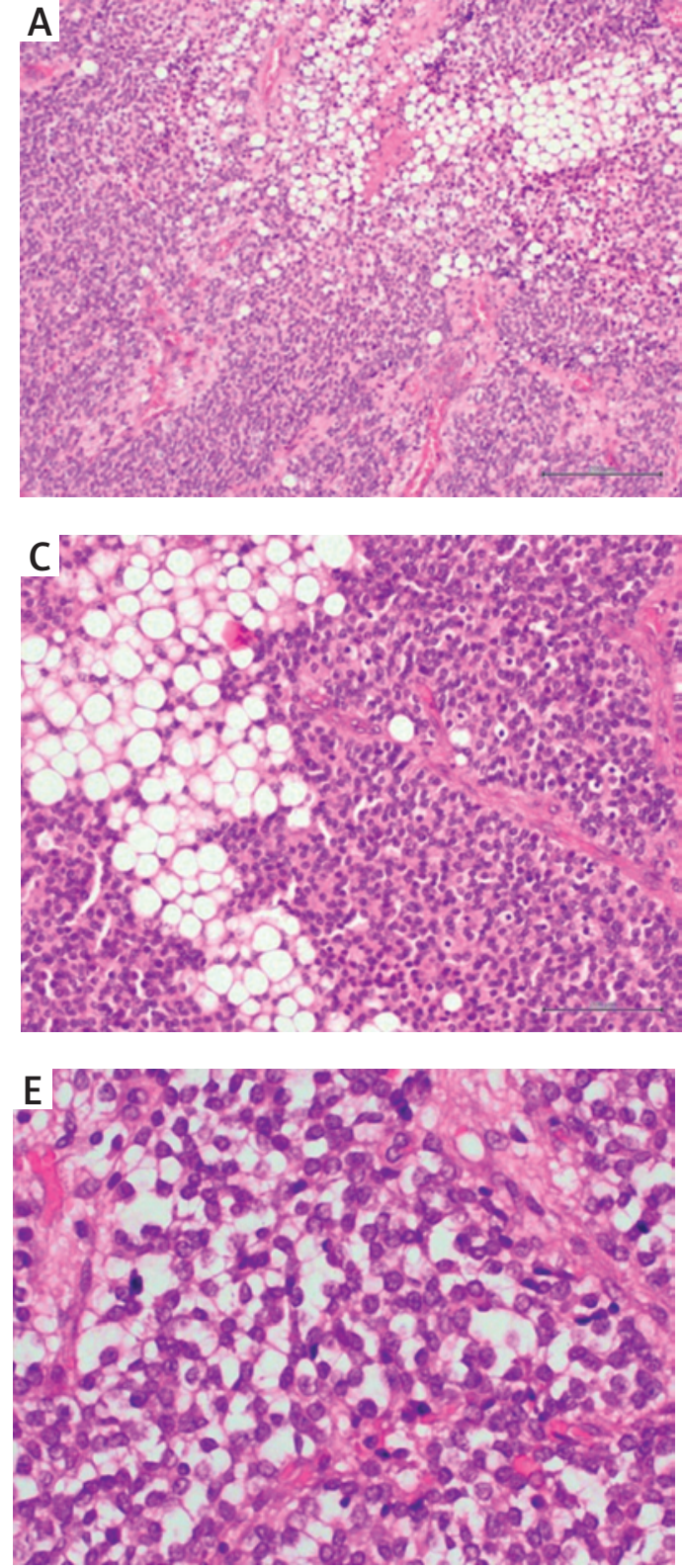
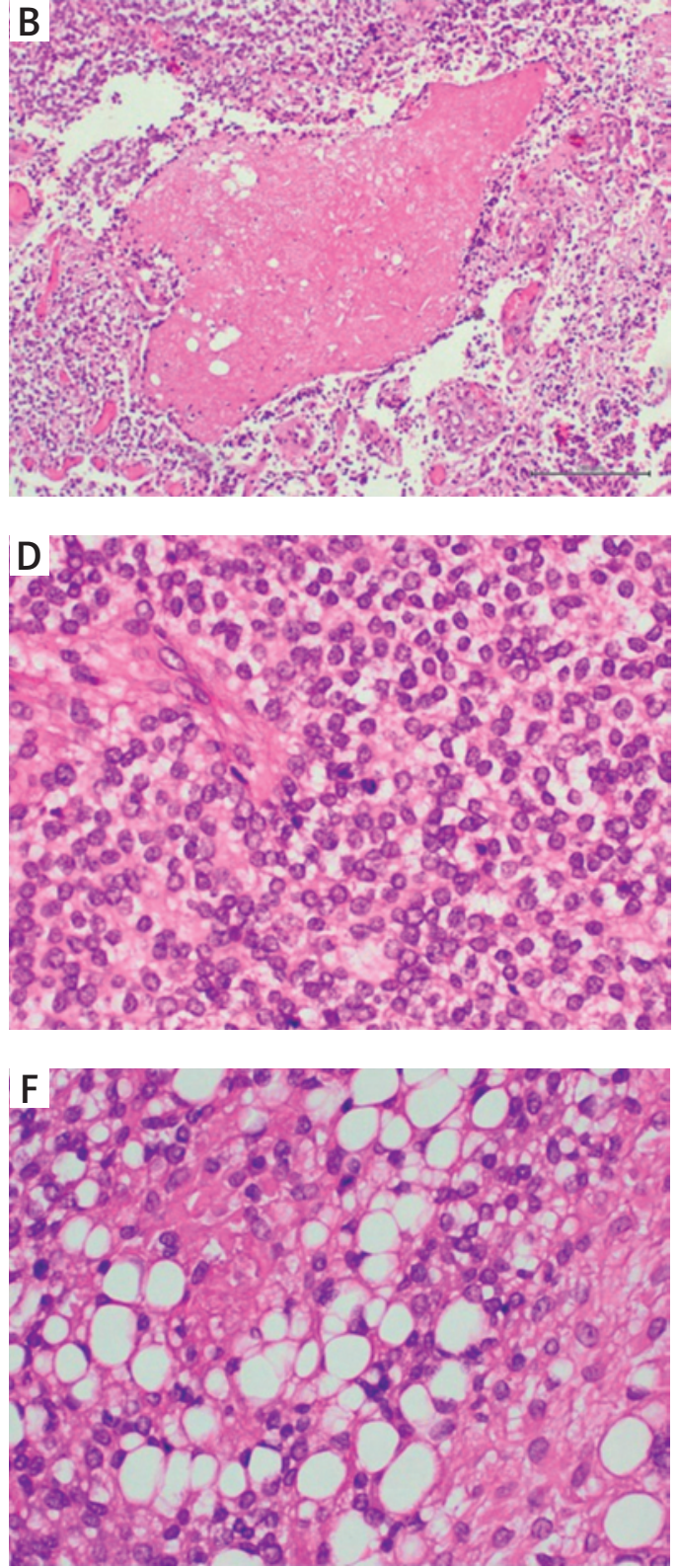

Fig. 2. Histopathological features of cerebellar liponeurocytoma in HE staining. A) Well-vascularized neoplastic tissue made of two components - small round cell population and lipomatous areas (100x). B) Focal necrosis and microvascular proliferations in lipomatous area (100x). C) Monotonous small neurocytic cell component with delicate rosettes intermixed with adipocytic focus (200x). D) Mitotically active round cells with regular nuclei embedded in scanty neuropil and with perivascular fibrillary matrix (400x). E) Neurocytic component showing cells with clear cytoplasm (400x). F) Lipomatous cells intermixed with numerous gemistocytic astrocytes producing fibrillary background (400x).

Magnetic resonance imaging $(\mathrm{MRI})$ showed a heterogeneous hypointense mass $(4 \times 3.5 \times 2.5 \mathrm{~cm})$ with hyperintense regions both in $\mathrm{T} 1$ and $\mathrm{T} 2$ with nonhomogeneous enhancement in the posterior fossa (Fig. 1). The tumor, arising from the cerebellar hemi- sphere, adhered widely to the dura and presented with minimal edema and signs of increased intracranial pressure due to hydrocephalus.

The patient underwent uncomplicated total resection of the lesion via suboccipital craniot- 
omy, confirmed by postoperative neuroimaging. The lobular soft and grayish mass suggestive of meningioma was revealed under the dura mater. The patient recovered from surgery without any complications. She was referred to the oncologist and neurosurgery outpatient department, which she attends on a regular basis. No additional treatment was introduced. Magnetic resonance imaging examinations every six months showed no evidence of recurrence, but only a small hyperintense focus in the post-resection region without contrast enhancement. The patient is free of symptoms 2 years after surgery.

\section{Neuropathological study}

The resected sample sent to the pathology department was irregular, brittle, beige and cream, measuring $4.2 \times 3.5 \times 2.5 \mathrm{~cm}$. The whole tumor tissue was routinely processed, examined and stained with HE. The selected sections underwent standard immunohistochemical proceedings according to the manufacturer recommendations. EnVision Systems (Dako) served for visualization, and diaminobenzidine as the chromogen. Appropriate positive and negative controls were carried out in parallel. We performed the following antibody detection: GFAP, synaptophysin, neurofilaments, NSE, S100, HMB-45, SMA, EMA, CD34, MyoD1, beta-catenin, HER2, Glut1, chromogranin, PAX5, vimentin, p53, desmin, CD99, Gli-1, Olig2, calretinin, Ki67 (DAKO, Abcam), and IDH1R132 (Dianova). Histochemical methods such as Masson trichrome and Gomori reticulin staining were also used.

The neuropathological examination (Fig. 2) showed a well-vascularized lobular tumor composed of two intermixed patterns made of distinct cellular populations. The blood vessels underlined tumor lobularity and presented segmental microvascular proliferation, including irregular glomeruloid forms. The predominant lobular component of the neoplasm was cell-rich, made of monotonous small round cells with scant pink cytoplasm and round to oval nuclei. A larger population with features of more advanced neuronal differentiation was also discernible. Delicate rosettes, rosette-like structures and areas of neuropil with glial and Rosenthal fibers and dispersed swollen neurites were visible. No evident cellular atypia was found. The mitotic index reached 15 mitoses per 10 high power fields,
Table I. Immunohistochemical profile of cerebellar liponeurocytoma

\begin{tabular}{|c|c|c|}
\hline Marker & $\begin{array}{l}\text { Small cell } \\
\text { component }\end{array}$ & $\begin{array}{c}\text { Glial-lipomatous } \\
\text { component }\end{array}$ \\
\hline GFAP & + & +++ \\
\hline Olig2 & + & ++ \\
\hline Synaptophysin, NSE & +++ & Negative \\
\hline Neurofilaments & + & Negative \\
\hline Calretinin, S-100 & +++ & +++ \\
\hline Chromogranin & ++ & Negative \\
\hline Vimentin & ++ & +++ \\
\hline GLUT1 & +++ & + \\
\hline Ki67 & Mean $12 \%$ & Mean 1\% \\
\hline
\end{tabular}

whilst the mean proliferation index Ki67 was about 12\% (Fig. 3A). In parallel, dispersed groups of apoptotic cells were encountered. The second histologic pattern of tumor was composed of irregular areas occupied by larger cells resembling adipocytes, intermingled with astrocytes mainly of gemistocytic type with plump nuclei and abundant eosinophilic cytoplasm. Gemistocytes were accumulated around blood vessels forming perivascular cuffs. In these areas, the mitotic as well as proliferation index was below $1 \%$. The fields of geographic necrosis and vascular thrombosis were more frequent in that component. The tumor-cerebellar tissue interface had an infiltrative character, accompanied by vascular reaction and small old hemorrhages.

Immunophenotype of this neoplasm was consistent with a glioneuronal tumor (Table I). The majority of the small neoplastic cells were NSE and synaptophysin-positive (Figs. 3B-C). In addition, chromogranin decorated some cells. Moreover, the majority of the neoplastic cells including a lipidized component were positive for vimentin, calretinin, Olig2 and S100 (Figs. 3D-E). Astrocytic and adipocyte-like cells, as well as some small cells, revealed GFAP reactivity (Figs. 3F-G). Neurofilaments were found in scattered single cells with ganglioid morphology, and in swollen perivascular axons. Strong GLUT-1 expression (Fig. 3H) was found in the endothelium of tumor microvessels. In neoplastic neuronal cells GLUT-1 reactivity was observed in a zonal pattern with increasing intensity and shifting from cytoplasmic to membranous staining in parallel to increasing distance from blood vessels as well as in perinecrotic regions. Beta-catenin was detected in a membranous/cytoplasmic pattern in both components. Staining for desmin, IDH-1, HMB-45, 

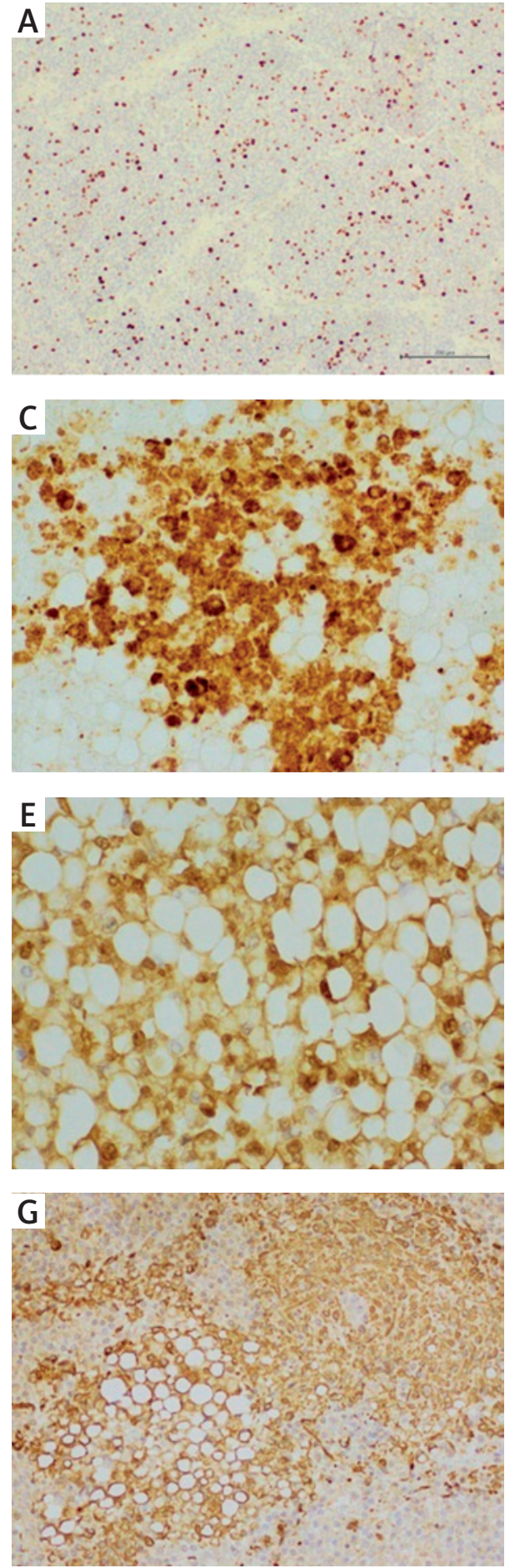
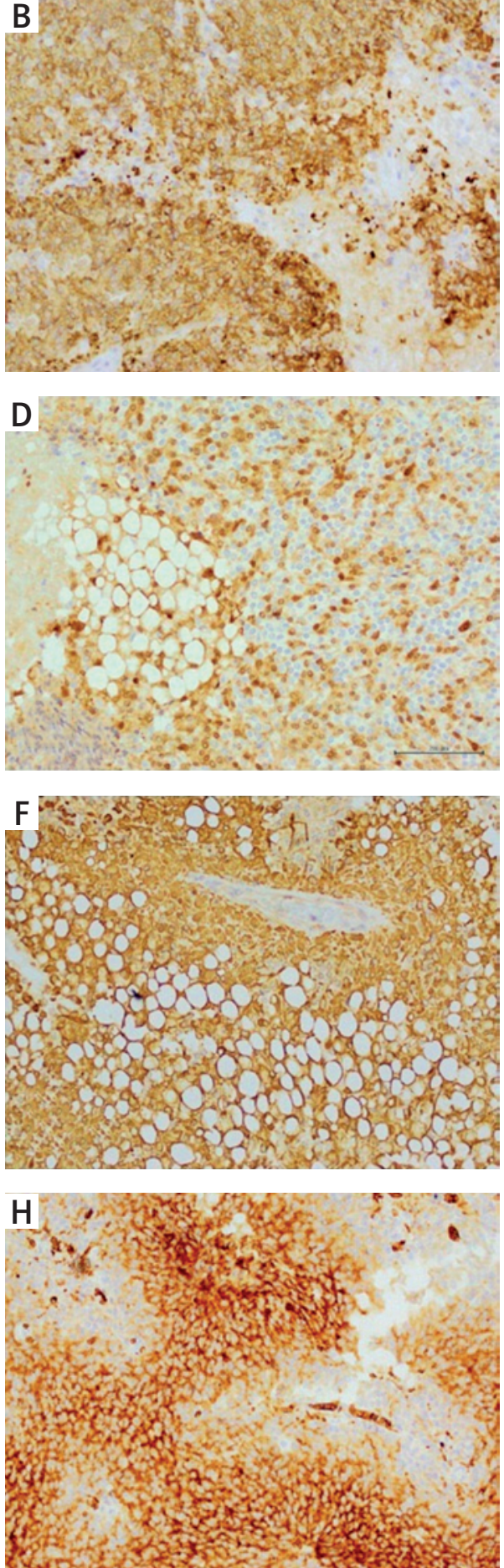

Fig. 3. Representative images of tumor immunophenotype. A) Ki-67 positive proliferating cells within the neurocytic areas of tumor (Ki-67, 40x). B) Diffuse synaptophysin expression within the tumor (synaptophysin, 200x). C) Synaptophysin-positive neural component and immunonegative lipomatous fields (synaptophysin, 400x). D) Irregular S100 immunoreactivity within the neoplastic tissue (S100, 200x). E) Strong S100 staining in lipomatous area (S100, 400x). F) Intense GFAP labeling of lipomatous and glial cell population (GFAP, 200x). G) GFAP-positive component and immunonegative neurocytic fields (GFAP, 100×). H) Prominent Glut1 reactivity within small neoplastic cell component and endothelial cells (Glut1, 100x). 
MyoD1, EMA, SMA, Gli-1, CD34, p53, Her2, CD99 and PAX5 was not observed in tumor cells. SMA underlined vascular network and microvascular proliferations.

The final histopathological diagnosis of cerebellar liponeurocytoma with atypical features, WHO grade II, was made.

\section{Discussion}

Cerebellar liponeurocytoma (CLNC), having several synonymous names, was initially called lipomatous medulloblastoma, because of some morphological similarities to that embryonal neoplasm. The other terms included medullocytoma and lipomatous glioneurocytoma [7,9,30] Importantly, the retrospective analysis of over 350 pediatric medulloblastomas [30] failed to identify any tumor with adipocytes. In 1998 [10] one case was called a lipidized mature neuroectodermal tumor of the cerebellum. In 2000, the WHO classification established the term 'cerebellar liponeurocytoma', defined as a rare neoplasm with consistent neuronal, variable astrocytic and focal lipomatous differentiation, located within the cerebellum, characterized by favorable prognosis $[1,14,16,17,20]$. However, longer clinical observations showed that cerebellar liponeurocytoma in almost half of the cases presents recurrences, so ultimately, in 2007 the neoplasm was reclassified as grade II [21]. The main location of this neoplasm is the cerebellum, but supratentorial and multifocal cases have also been reported $[5,13,19,25]$, as well as intraventricular examples $[17,20]$, and tumors with extracranial extension [3].

Cerebellar liponeurocytoma occurs in adults with the mean age of 50 years, with no sex predilection. Usually patients present with headache, focal neurologic deficits or obstructive hydrocephalus. On CT scans CLNC appears as a hypo- or isodense tumor with focal areas of marked hypoattenuation corresponding to fat density [23]. Magnetic resonance imaging is quite characteristic and helps to distinguish the neoplasm from others in this location. In general, these tumors are usually heterogeneous, well circumscribed with lipid content [12]. On T1-weighted images CLNC is iso- or hypointense, with patchy areas of hyperintensity, while on T2-weighted images it is slightly hyperintense to the surrounding brain. Contrast enhancement may be low and often is heterogeneous, while peritumoral edema is minimal or absent $[12,23]$.

Histopathologically, the neoplasm is composed of round to oval relatively small cells with ill-defined borders admixed with areas of adipocyte-like cells. Importantly, in recurrences, the lipidized component may be very limited or absent. Entities which enter the differential diagnosis with CLNC include rare cases of metaplastic meningiomas with adipocytic component, pleomorphic xanthoastrocytomas, glioblastomas, oligodendroglioma and neurocytomas with lipid component [18]. However, in these entities, multivacuolar or foamy lipidization is more often seen. The important and basic tool for differential diagnosis in liponeurocytoma is immunophenotyping, where small and oligodendroglia-like cells show neuronal differentiation (synaptophysin, neuron-specific enolase). Lipidized cells are of neuroepithelial origin too. In CLNC, astrocytic differentiation identified by GFAP and Olig2 staining is seen only focally. Single cases with positivity of myoid or ependymal $[10,15,17]$ markers have also been described. Typically, the proliferation index measured with Ki67 is usually low, ranging between 1 and $6 \%$. To date only a few cases of CLNC with atypical histological features comprising prominent microvascular proliferation and/or necrosis have been reported $[6,8,24,27]$. These cases also presented a high proliferation index and areas of hemorrhages.

Our patient is one of the oldest reported. Moreover, she was previously successfully treated for two different neoplasms: colon cancer and a spinal schwannoma. Clinically the patient was suspected of metastatic tumor, but the radiological picture suggested meningioma. Histologically it was liponeurocytoma composed of neurocytic and astrocytic components. The components revealed different immunophenotype, sharing S100, calretinin and vimentin positivity. The proliferation index of the tumor was relatively high, up to $12 \%$ in the component with neural differentiation. Interestingly, lipidized areas contained gemistocytic islands and revealed mainly an astrocytic immunoprofile that was not active mitotically. Our case contained unusual coagulative geographic necroses within the lipidized component and multifocal microvascular proliferation with glomeruloid forms. The neural part revealed small necrotic foci. Vascular proliferation was more intense in the borders of necrosis and in the infiltrative front. 
Microvascular proliferation and necrosis can be observed in many brain tumors, being considered as hallmarks of histological malignancy and unfavorable prognosis in diffuse gliomas. "Classical" coagulative necrosis in some cases has no prognostic significance, as in ependymomas and pilocytic astrocytomas. Conversely, the presence of necrosis upgrades diffuse gliomas, pleomorphic xanthoastrocytoma, and meningiomas. Moreover, our tumor presented prominent glucose transporter 1 (GLUT-1) reactivity, which shifted from cytoplasmic in cells lying away from microvessels to membranous in perinecrotic areas. Tumor cells around microvessels were completely immunonegative for the transporter. GLUT-1 expression is induced under hypoxic conditions. This phenomenon occurs in various types of malignancy including high-grade gliomas, where it mediates increased glucose uptake, being also associated with a worse clinical outcome [29]. Observed expression of GLUT-1 together with atypical histological features can support the higher histological grade in our case.

Cerebellar liponeurocytoma is classified as a neoplasm with advanced neuronal/neurocytic differentiation and focal lipoma-like changes [22]. Not only the neural component, but also some fat-laden cells show positive staining for neuronal markers in our case. Genetic studies $[3,21]$ showed that TP53 mutations were found in $20 \%$ of these tumors, but no other alterations such as iso 17q, chromosome 6 monosomy or mutations of PTCH, APC or beta-catenin. Thus, CLNC is different from medulloblastoma and more similar to neurocytoma but with a distinct genetic pathway due to the presence of TP53 mutations [14]. Moreover, Hirokazu et al. [32] reported the absence of both chromosome $1 p / 19 q$ loss and IDH1 mutation, which might be useful in differential diagnosis of oligodendroglioma [4]. Our tumor showed neither immunohistochemical features of p53 and IDH1 alterations nor SHH and $\beta$-catenin activation. These findings helped in differential diagnosis and excluded by immunohistochemical molecular surrogates common genetic changes in CNS tumors.

The dynamics of the initial lesional growth in our patient seems to be rather high, because one year before onset of cerebellar symptoms she had negative control oncological neuroimaging. Two years after resection, the patient is free of symptoms and radiologic recurrence. Nearly half of patients with cerebellar liponeurocytomas suffer recurrences [20], and a few cases have been reported as clinically aggressive $[16,24]$. The basic therapeutic method in the described tumor is total neurosurgical resection. Treatment options have not yet been clearly established due to rarity of the lesion and lack of understanding of its biological behavior. It is unclear whether radiation should be given immediately after surgical resection or be reserved for recurrence [23].

The unusual atypical histological features of this cerebellar liponeurocytoma include the high proliferation index, fields of geographic necrosis, and multifocal microvascular proliferation, as well as the prominent astrocytic component. Perhaps due to the composite histology spectrum with different potential locations of the lesion described in the literature, the term 'lipomatous glioneuronal tumor' is more appropriate for this type of neoplasm.

\section{Disclosure}

Authors report no conflict of interest.

\section{References}

1. Aker FV, Ozkara S, Eren P, Peker O, Armağan S, Hakan T. Cerebellar liponeurocytoma/lipidized medulloblastoma. J Neurooncol 2005; 71: 53-59.

2. Bechtel JT, Patton JM, Takei Y. Mixed mesenchymal and neuroectodermal tumor of the cerebellum. Acta Neuropathol 1978; 41: 261-263.

3. Ben Nsir A, Ben Said I, Hammami N, Sebai R, Jemel H. Cerebellar liponeurocytoma with extracranial extension: case report. Neurol Neurochir Pol 2014; 48: 458-462.

4. Capper D, Reuss D, Schittenhelm J, Hartmann C, Bremer J, Sahm F, Harter PN, Jeibmann A, von Deimling A. Mutation-specific IDH1 antibody differentiates oligodendrogliomas and oligoastrocytomas from other brain tumors with oligodendroglioma-like morphology. Acta Neuropathol 2011; 121: 241-252.

5. Chakraborti S, Mahadevan A, Govindan A, Yasha TC, Santosh V, Kovoor JM, Ramamurthi R, Alapatt JP, Hedge T, Shankar SK. Supratentorial and cerebellar liponeurocytomas: report of four cases with review of literature. J Neurooncol 2011; 103: 121-127.

6. Châtillon CE, Guiot MC, Roberge D, Leblanc R. Cerebellar liponeurocytoma with high proliferation index: treatment options. Can J Neurol Sci 2009; 36: 658-661.

7. Chimelli L, Hahn MD, Budka H. Lipomatous differentiation in a medulloblastoma. Acta Neuropathol 1991; 81: 471-473.

8. Chung SB, Suh YL, Lee Jl. Cerebellar liponeurocytoma with an unusually aggressive histopathology: case report and review of the literature. J Korean Neurosurg Soc 2012; 52: 250-253.

9. Ellison DW, Zygmunt SC, Weller RO. Neurocytoma/lipoma (neurolipocytoma) of the cerebellum. Neuropathol Appl Neurobiol 1993; 19: 95-98. 
10. González-Cámpora R, Weller RO. Lipidized mature neuroectodermal tumour of the cerebellum with myoid differentiation. Neuropathol Appl Neurobiol 1998; 24: 397-402.

11. Gottberg E, Ziad F. Liponeurocytoma with unusual features. Pathology 2016; 48: 125.

12. Guan JT, Geng YQ, Cheng Y, Guo YL, Wu RH. Magnetic resonance imaging of cerebellar liponeurocytoma. A case report and review of the literature. Neuroradiol I 2012; 25: 331-336.

13. Gupta K, Salunke P, Kalra I, Vasishta RK. Central liponeurocytoma: case report and review of literature. Clin Neuropathol 2011; 30: 80-85.

14. Horstmann S, Perry A, Reifenberger G, Giangaspero F, Huang H, Hara A, Masuoka J, Rainov NG, Bergmann M, Heppner FL, Brandner S, Chimelli L, Montagna N, Jackson T, Davis DG, Markesbery WR, Ellison DW, Weller RO, Taddei GL, Conti R, Del Bigio MR, González-Cámpora R, Radhakrishnan VV, Söylemezoglu F, Uro-Coste E, Qian J, Kleihues P, Ohgaki H. Genetic and expression profiles of cerebellar liponeurocytomas. Brain Pathol 2004; 14: 281-289.

15. Hortobágyi T, Bódi I, Lantos PL. Adult cerebellar liponeurocytoma with predominant pilocytic pattern and myoid differentiation. Neuropathol Appl Neurobiol 2007; 33: 121-125.

16. Jenkinson MD, Bosma JJ, Du Plessis D, Ohgaki H, Kleihues P, Warnke P, Rainov NG. Cerebellar liponeurocytoma with an unusually aggressive clinical course: case report. Neurosurgery 2003; 53: 1425-1427.

17. Jouvet A, Lellouch-Tubiana A, Boddaert N, Zerah M, Champier J, Fèvre-Montange $M$. Fourth ventricle neurocytoma with lipomatous and ependymal differentiation. Acta Neuropathol 2005; 109: 346-351.

18. Kakkar A, Sable M, Suri V, Sarkar C, Garg A, Satyarthee GD, Sharma MC. Cerebellar Liponeurocytoma, an Unusual Tumor of the Central Nervous System - Ultrastructural Examination. Ultrastruct Pathol 2015; 39: 419-423.

19. Karabagli P, Sav A, Pamir N. Does "cerebellar liponeurocytoma" always reflect an expected site? An unusual case with a review of the literature. Folia Neuropathol 2014; 52: 101-105.

20. Kuchelmeister K, Nestler U, Siekmann R, Schachenmayr W. Liponeurocytoma of the left lateral ventricle - case report and review of the literature. Clin Neuropathol 2006; 25: 86-94.

21. Louis DN, Ohgaki H, Wiestler OD, Cavenee WK, Burger PC, Jouvet A, Scheithauer BW, Kleihues P. The 2007 WHO classification of tumours of the central nervous system. Acta Neuropathol 2007; 114: 97-109.

22. Louis DN, Ohgaki H, Wiestler OD, Cavenee WK. WHO Classification of Tumours of the Central Nervous System, Revised. Fourth Edition. Volume 1. IARC, 2016.

23. Nishimoto T, Kaya B. Cerebellar liponeurocytoma. Arch Pathol Lab Med 2012; 136: 965-969.

24. Pasquale G, Maria BA, Vania P, Gastone P, Nicola DL. Cerebellar liponeurocytoma: an updated follow-up of a case presenting histopathological and clinically aggressive features. Neurol India 2009; 57: 194-196.

25. Pelz D, Khezri N, Mainprize T, Phan N, Keith J, Bilbao J, Aviv RI, Tsao M, Symons SP. Multifocal cerebellar liponeurocytoma. Can J Neurol Sci 2013; 40: 870-872.
26. Pikis S, Fellig Y, Margolin E. Cerebellar liponeurocytoma in two siblings suggests a possible familial predisposition. J Clin Neurosci 2016; 32: 154-156.

27. Radke J, Gehlhaar C, Lenze D, Capper D, Bock A, Heppner FL, Jödicke $A$, Koch $A$. The evolution of the anaplastic cerebellar liponeurocytoma: case report and review of the literature. Clin Neuropathol 2015; 34: 19-25.

28. Rickert C, Riemenschneider M, Schachenmayr W, Richter H, Bocjhorn J, Reinferberger G, Paulus W. Glioblastoma with adipocyte-like tumor cell differentiation - histological and molecular features of a rare differentiation pattern. Brain Pathol 2009; 19: 431-438.

29. Shibuya K, Okada M, Suzuki S, Seino M, Seino S, Takeda H, Kitanaka C. Targeting the facilitative glucose transporter GLUT-1 inhibits the self-reneval and tumor-initiating capacity of stem cells. Oncotarget 2015; 6: 651-661.

30. Soylemezoglu F, Soffer D, Onol B, Schwechheimer K, Kleihues P. Lipomatous medulloblastoma in adults. A distinct clinicopathological entity. Am J Surg Pathol 1996; 20: 413-418.

31. Takami H, Mukasa A, Ikemura M, Shibahara J, Takahashi M, Momose T, Saito N. Findings from positron emission tomography and genetic analyses for cerebellar liponeurocytoma. Brain Tumor Pathol 2015; 32: 210-215.

32. Wang K, Ni M, Wang L, Jia G, Wu Z, Zhang L, Zhang J. Cerebellar liponeurocytoma: a case report and review of the literature. Oncol Lett 2016; 11: 1061-1064.

33. Wolf A, Alghefari H, Krivosheya D, Staudt MD, Bowden G, Macdonald DR, Goobie S, Ramsay D, Hebb MO. Cerebellar liponeurocytoma: a rare intracranial tumor with possible familial predisposition. Case report. J Neurosurg 2016; 125: 57-61. 